\title{
Tuberculosis in Chronic Obstructive Pulmonary Disease
}

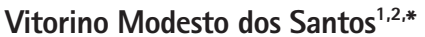 \\ ${ }^{1}$ Medical Course, Catholic University of Brasília, Brasilia-DF, Brazil \\ ${ }^{2}$ Department of Internal Medicine, Armed Forces Hospital, Brasilia-DF, Brazil
}

\section{Dear Editor}

I read with great interest the article by Park et al. ${ }^{1}$ in which they evaluated the prevalence, awareness, and main risk factors of chronic obstructive pulmonary disease (COPD) in 8,969 Korean adults, taking into account sex, age, smoking history, social and nutrition status, in addition to asthma and tuberculosis history. It is worthy of note, however, that it was difficult to exclude the possibility of asthma overlapping with COPD. More so, tuberculosis was classified as suspected, active or inactive by chest radiography findings. ${ }^{1)}$ Other recent studies have revealed that individuals with COPD are frequently affected by one or more comorbidities, and these conditions often evolve unrecognized during the aging process. ${ }^{2)}$ Infection; cancer; and metabolic, cardiac, and neuropsychiatric disorders may occur concurrently, and may therefore result in additional hospital care costs, poorer quality of life, and worse outcomes overall. ${ }^{2)}$ Pneumonia and mycobacterial infections may increase morbidity and mortality rates related to acute exacerbations of COPD, and unsuspected tuberculosis is a major concern. ${ }^{3)}$ Both corticosteroids and levofloxacin are commonly utilized to control exacerbations of pulmonary symptoms, but use of these drugs may led to diagnostic challenges in this group of patients. ${ }^{3)}$ The use of fluoroquinolone can hinder the diagnosis of tuberculosis in culture media, and this severe disease may therefore develop unrecognized, resulting in poor prognoses in immunosuppressed patients. ${ }^{3)}$ For example, unsuspected active tuberculosis occurred in a 92-year-old Brazilian woman who was a heavy smoker with repeated ex- acerbations of COPD and who was treated with inhaled corticosteroids and levofloxacin. ${ }^{3)}$ Radiography and computed tomography of the chest did not suggest active tuberculosis, but subsequent cultures revealed $M y$ cobacterium tuberculosis while the patient was prescribed piperacillin-tazobactam instead of fluoroquinolone. Levofloxacin, a second-line drug for tuberculosis, may cause diagnostic pitfalls in COPD. ${ }^{3)}$ In Korea and in Brazil, elderly smokers with comorbidities are commonly affected by COPD. Moreover, tuberculosis constitutes a major public health problem in the whole world, mainly in developing countries.

\section{CONFLICT OF INTEREST}

No potential conflict of interest relevant to this article was reported.

\section{REFERENCES}

1. Park H, Jung SY, Lee K, Bae WK, Lee K, Han JS, et al. Prevalence of chronic obstructive lung disease in Korea using data from the fifth Korea National Health and Nutrition Examination Survey. Korean J Fam Med 2015;36:128-34.

2. Milkowska-Dymanowska J, Bialas AJ, Zalewska-Janowska A, Gorski P, Piotrowski WJ. Underrecognized comorbidities of chronic obstructive pulmonary disease. Int J Chron Obstruct Pulmon Dis 2015;10:1331-41.

3. Modesto dos Santos V, Martins RR, Fachinelli LR, Araujo MC, dos Santos UM, Ribeiro KD. Unsuspected tuberculosis in COPD and use of levofloxacin: diagnostic challenges. Infez Med 2014; 22:309-12. 